\title{
Monolateral external fixation for the progressive correction of neurological spastic knee flexion contracture in children
}

\author{
Pedro Gutiérrez Carbonell · Jose Valiente Valero • \\ Pedro Doménech Fernández · Javier Roca Vicente-Franqueira
}

Received: 11 July 2007 / Accepted: 24 November 2007 / Published online: 4 December 2007

(C) Springer-Verlag 2007

\begin{abstract}
The purpose of this study was to report the results of the surgical treatment of spastic knee flexion contracture using tenotomy and progressive correction by external fixator-distractor devices. The study design involved a prospective observational study of 16 knees in nine patients with spastic flexion contracture greater than $30^{\circ}$. Treatment was indicated for both ambulatory and nonambulatory patients; and, in the latter group when sitting or personal hygiene was compromised. The average age was 11.6 years (range 10-17). Five of the patients were male and four female. There was one case of hemiplegia $(11.1 \%)$, two cases of paraplegia (22.2\%), and six cases of quadriplegia $(66.7 \%)$. Six patients retained some walking capacity, while three had none. In all cases, distal lengthening of the hamstrings was carried out. A monolateral fixator with a gradual correction device was applied for a period of 4.8 weeks. The average follow-up was 26.6 months. The preoperative straight-leg raise was $55^{\circ}$. The popliteal angle was $58^{\circ}$ preoperatively (range $30-80^{\circ}$ ), $8.5^{\circ}$ on removal of the fixator, and $20^{\circ}$ at the end of the follow-up. Complications: There were no superficial or deep infections, and no fractures or distal sensory-motor alterations. There was one case of arthrodiatasis of the knee (6.3\%) which was resolved when the fixator was removed,
\end{abstract}

P. G. Carbonell · J. V. Valero · P. D. Fernández .

J. R. Vicente-Franqueira

Unit of Pediatric Orthopedic Surgery,

General Hospital of Alicante,

Department of Pathology and Surgery,

University of Miguel Hernández,

Elche, Alicante, Spain

P. G. Carbonell ( $\square)$

Paraje Ledua E-25, 03660 Novelda, Alicante, Spain

e-mail: gutierrez_ped@gva.es and 11 cases of pin-track infection (68.7\%) which were resolved with local care and oral antibiotics. To conclude, spastic knee flexion contracture can be treated gradually with monolateral external fixator with distraction devices, and with distraction modules which prevent acute stretching of the posterior neurovascular structures of the knee.

Keywords Knee flexion - Neurological knee flexion · Distal lengthening hamstrings in knee flexion contracture . External fixator - Gradual correction

\section{Introduction}

Cerebral palsy is the most frequent neuromuscular dysfunction in childhood. Its incidence, in absolute terms, has been almost constant over the last few decades, affecting 2.2 and $2.7 \times 10^{3}$ liveborns, but in recent years, the proportion of the types of neurological affectation has changed [1-3]. The diplegic and especially quadriplegic types of cerebral palsy have experienced an increase of between 50 and $150 \%$, and this is mainly due to the current improvement in postnatal resuscitation methods which have produced a $30 \%$ increase in the survival rate of preterm babies with low birthweight [2, 4, 5]. Children under 28-30 weeks of gestation and weighing between 1,300 and $1,800 \mathrm{~g}$ on delivery represent the new population at risk of cerebral palsy. Sixty-five percent of these cases are spastic type, with $25 \%$ being athetoid and the rest mixed [5-7]. Hyperexcitability of the motorneurone stretch reflex is a common feature of spastic muscles and therefore, if and when a patient begins to walk, he does so in a crouching position with flexion of the hips and knees greater than $30^{\circ}$ and with feet in the equinus position, the result of weakness of the quadriceps and spasticity of the hamstrings and 
gastrocnemius [8-10]. The most important problems in these children are knee flexion with a crouch gait in those who are ambulatory, and access for personal hygiene in those who are not $[2,7,11-15]$. We describe the results of surgical treatment of spastic knee flexion contractures, supplementing fractional lengthening of muscles and/or tendons with progressive correction (in the immediate postoperative period) through a unilateral external fixator, which uses a hinge system and a distractor device.

\section{Materials and methods}

Between 2000 and 2004, we treated 16 spastic knee flexion contractures in nine patients. ${ }^{1}$ In all cases, the following were measured and performed: (a) the popliteal angle (Holt's angle) $^{1}$ [2, 4, 9, 10, 16-18], (b) the straight-leg raising angle ${ }^{2}$, (c) the Duncan-Ely test ${ }^{3}$, and (d) the mobility arch of the knee $[2,7,11,12,19]$. The inclusion criteria were:

(1) Spastic patients with hemiplegia (one patient, $11.1 \%$ ), paraplegia (two patients, $22.2 \%$ ), or quadriplegia (six patients, $66.7 \%$ ),

(2) If the straight-leg raising angle was less than $50^{\circ}$,

(3) A popliteal angle equal ${ }^{1}$ to or greater than $30^{\circ}$,

(4) A negative Duncan-Ely test,

(5) Standing difficulties in ambulatory patients, or chairto-bed transfer difficulties for the nonambulatory patients,

(6) Sitting position and personal hygiene care difficulties in non-ambulant patients, and

(7) At least 1 year of medical and/or rehabilitation treatment for the flexion contracture without success $[4,9,14,15,18,20]$.

Children were classified according to functional levels [16, 21] as: community ambulators who were indoor and outdoor ambulants, with or without the help of a crutch (two patients, 22.2\%); household ambulators (two patients, $22.2 \%$ ) who were non-functional ambulators, walking being possible only with external braces and usually done during rehabilitation therapy (two patients, 22.2\%); and

\footnotetext{
1 Angle between the posterior thigh and posterior calf. The patient is in supine position with the contralateral hip in extension, the tested limb is flexed to $90^{\circ}$ at the hip and the knee is extended passively. This angle determines the degree of hamstring spasticity or contracture.

${ }^{2}$ Patient in supine position, knee should be in incomplete extension. Straight-leg raising angle is the angle between lower limb and examination table.

${ }^{3}$ The patient is in prone position and then flexes the knee to $90^{\circ}$. If there is spasticity in the quadriceps and especially of the rectus femoris, the ipsilateral buttock will rise from the table.
}

non-ambulators or wheelchair-bound (three cases, 33.3\%). Out of the nine patients, four $(44.4 \%)$ retained ambulatory capacity and five $(55.6 \%)$ had none or were very limited. The average age was 11.6 (range 10-17 years), 10.5 for males and 11.0 for females. Five of the nine patients were male $(55.6 \%)$ and four female $(44.4 \%)$. The procedure was bilateral and simultaneous in seven patients $(77.8 \%)$ and unilateral in two (22.2\%). The average follow-up was 26.6 months (range 8-45 months). The fixator was removed at an average of 4.8 weeks (range 4-8 weeks).

A mobility arch of the knee between $0^{\circ}$ (full extension) and $130^{\circ}$ (maximum flexion) was considered normal. Extension deficits were noted in negative degrees. Measurements were taken using a goniometer calibrated in two steps. The average straight-leg raising angle was $55 \pm 4.5^{\circ}$ (range $30-66^{\circ}$ ). The popliteal angle was $57.7 \pm 3.5^{\circ}$ (range $30-80^{\circ}$ ) and the average preoperative mobility arch was between 50 and $100^{\circ}$ flexion. Descriptive statistic and non-parametric tests (Wilcoxon) were used, with a $P<0.05$ level of significance [22].

\section{Surgical technique}

The procedure was carried out in two stages:

(1) Prone position: Two incisions, medial and lateral, were made in seven patients $(77.8 \%)$ and an italic S-shaped incision in the other two (22.2\%). Z-lengthening of the semitendinosus and gracilis tendons was performed. The biceps and semimembranosus muscles were lengthened by circumferentially dividing the tendinous aponeurosis. The posterior portion of the ilio-tibial band was also divided. If extension of the knee had not improved sufficiently by then, lateral and medial heads of gastrocnemius were also divided. The posterior capsule was not divided $[2,9,12,14$, 16, 20, 23, 24].

(2) Supine position: Placement of a monolateral fixator (LRS system, Orthofix ${ }^{\circledR}$, Verona, Italy) with screws in the femur and tibia and an intervening hinge positioned so as to coincide with the instantaneous centre of rotation for the knee. Two compressor-distractor devices, the first one medium-sized and in the dorsal position, and a second, large and in the volar position, were spanned across the hinge by anchoring to the proximal and distal rails on special clamps (Figs. 1,2). Placement of the external fixator was performed with fluoroscopic control. A $1.5 \mathrm{~mm} \mathrm{~K}$-wire was inserted through the central orifice of the fixator's rotation hinge to coincide with the centre of rotation of the knee which, according to anatomical and biomechanical studies [25-29], is located at the intersection of 
the posterior femoral cortex and the intercondylar notch, and in the frontal plane passes through the origins of the medial collateral and lateral collateral ligaments (Fig. 3). After situating this rotation centre, both the proximal femoral and distal tibial rails were each fixed using two threaded half-pins through clamps (Fig. 4). The accuracy of our efforts was verified in vitro using the concentric circles method $[27,28,30,31]$. Our calculations revealed that a $1 \mathrm{~mm}$ distraction of the posterior compressor-distractor device produces $1-2^{\circ}$ gradual knee extension. The estimate of the average correction period was 15.7 days. The fixator hinge was locked at $-30^{\circ}$ in the immediate postoperative period and up to $4-5 \mathrm{~mm}$ of distraction was applied to the anterior compressordistractor device at a rate of $1 \mathrm{~mm} /$ day. This was done after unlocking the tibial clamp on the tibial rail, thereby minimizing any potential crushing of the articular cartilage during the subsequent angular correction. When this distraction was accomplished, the tibial clamp was locked and distraction now applied to the posterior unit (at $1 \mathrm{~mm} / 8 \mathrm{~h}$ ), with the femoral clamp on the rail and hinge unlocked, until complete extension of the knee was achieved. This usually occurred after a 14-18 days period. The fixator and the half pins were removed as an outpatient procedure within a $4-8$ week period after surgery. No cast immobilization of the knee was used. The rehabilitation process was initiated immediately after removal of the fixator. We used nocturnal postural orthoses for a period of 2-3 months.

Results were classified as excellent or good, average, and poor, based on degree of flexion, degree of loss of the range of motion of the knee, and the occurrence of complications at final clinical evaluation $[25,27]$.

\section{Results}

The postoperative straight-leg raising angle averaged $75^{\circ}$ (range $60-80^{\circ}$ ). This increase was not statistically significant although there was no loss during the follow-up period. The average extension after the removal of the fixator was $-8.5^{\circ}$ (range 0 to $-15^{\circ}$ ), and $-19.6^{\circ}$ (range -10 to $-25^{\circ}$ ) at the end of the monitoring period. This difference between flexion contracture after the removal of the external fixator and at the end of monitoring period was significant $(P<0.001)$. The range of motion of the knee at the end of the follow-up period was $90^{\circ}$ (range $80-110^{\circ}$ ), with no significant decrease in comparison to preoperative values.

No soft tissue releases to correct hip flexion (tenotomy or rectus femoris transfers) or equinus deformity of the feet (Achilles' tendon lengthening) were carried out coincidental to this surgery. At follow-up, the Duncan-Ely test remained negative at $110^{\circ}$ degrees of knee flexion and after the follow-up period, except in the case of one nonambulant quadriplegic who needed a rectus femoris lenthening 15 months after the knee flexion procedure.
Fig. 1 a LRS-monolateral external fixator system rails; b special clamp that blocks the compressor-distractor devices; $\mathbf{c}, \mathbf{d}$ lateral and superior view of special clamps threading inside rail system
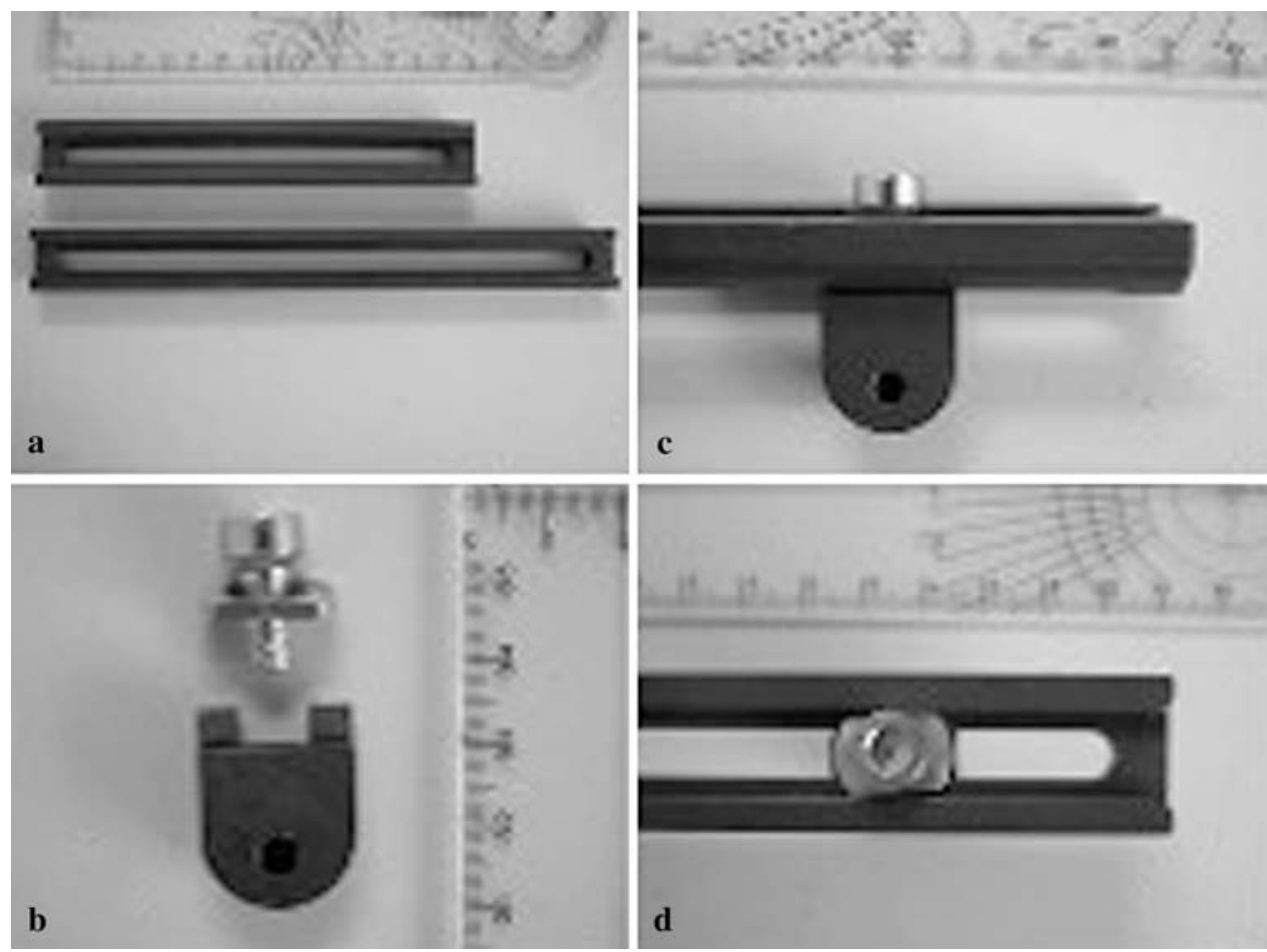
Fig. 2 a, b Lateral and superior view of rotation hinge; c compressor-distractor devices (short and large)
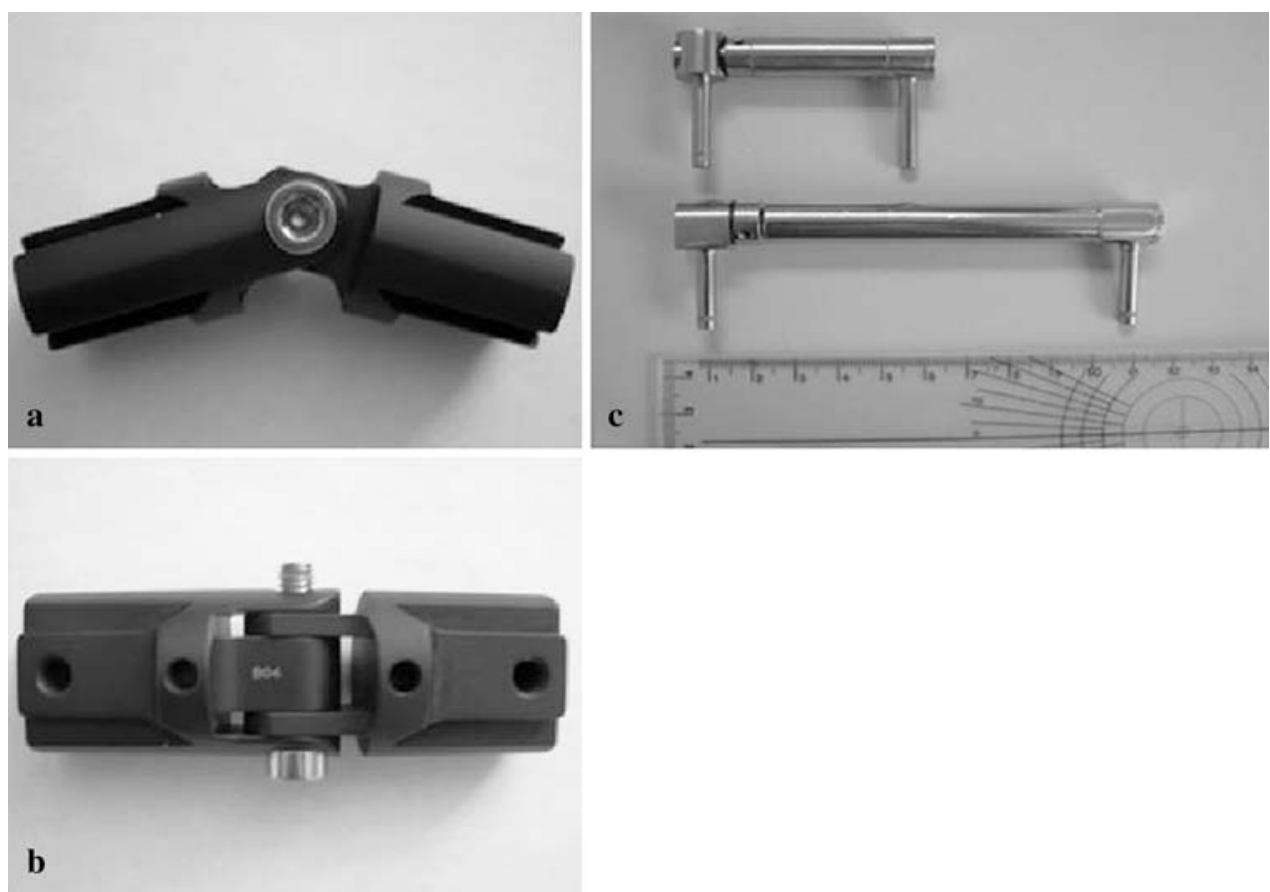

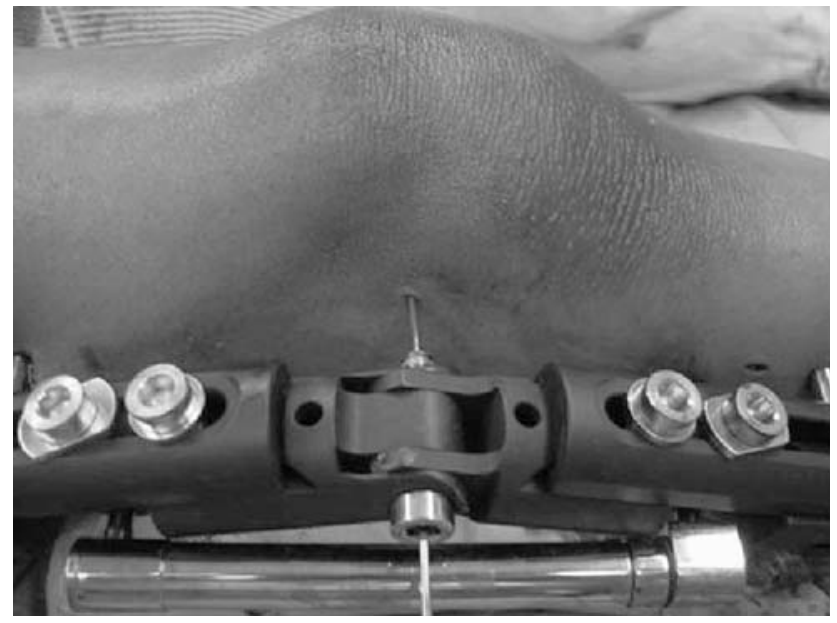

Fig. 3 Provisional placement of the guided K-pin, that allows the setting of the hinge in the knee's rotation center

The fixator was in place for an average of 4.8 weeks (range 4-8 weeks). The ability of children who could walk before surgery was unchanged after the procedure. Improvement of at least one level of function, according to the classification used, was observed in $89 \%$ of the cases. Four patients (33.3\%) achieved outdoor community ambulatory status, two (22.2\%) became household ambulant, and during the rehabilitation treatment, two (22.2\%) progressed from wheelchair-bound to ambulant with the help of two crutches and orthoses. One patient (11.1\%) remained wheelchair-bound. This patient did not improve his level of function but experienced a better sitting position in the wheelchair as well as a higher degree of comfort for postural changes and hygienic care. According to the classification system we used, the following classification results were obtained: five patients $(55.5 \%)$ yielded excellent and good results, three patients $(33.4 \%)$ yielded average results, and one patient $(11.1 \%)$ yielded unsatisfactory results.

One major complication occurred: there was a tibiofemoral arthrodiatasis, which arose due to a technical error when the hinge that allowed for progressive extension was locked, causing excessive joint distraction. This complication was resolved by simply unlocking the hinge and left the patient without long-term clinical or neurological sequelae. As regards minor complications, genu recurvatum occurred in four cases (25\%) but in no case exceeded $10^{\circ}$. There were pin track infections in 11 cases $(68.7 \%)$, which were resolved by pin site care and, in three cases (18.7\%), by including oral antibiotic therapy. No vascular or nerve injuries, compartmental syndromes, physeal injuries, tibial or femoral fractures were observed.

\section{Discussion}

Spastic knee flexion contractures significantly alter the kinematics of walking and dramatically reduce the possibility of ambulation. It also alters the ease of postural hygienic care of non-ambulant patients [7, 9, 11, 14, 18]. Alterations in the duration of the stages of ambulation and a swing phase that challenges balance are common kinematics of spastic patients. This makes it difficult for them to stand. When walking, they do so with decreasing 
Fig. 4 a, b "In Vitro" and c "In Vivo" mobility of the hinge, when elongation-distraction is applied to the compressordistractor device
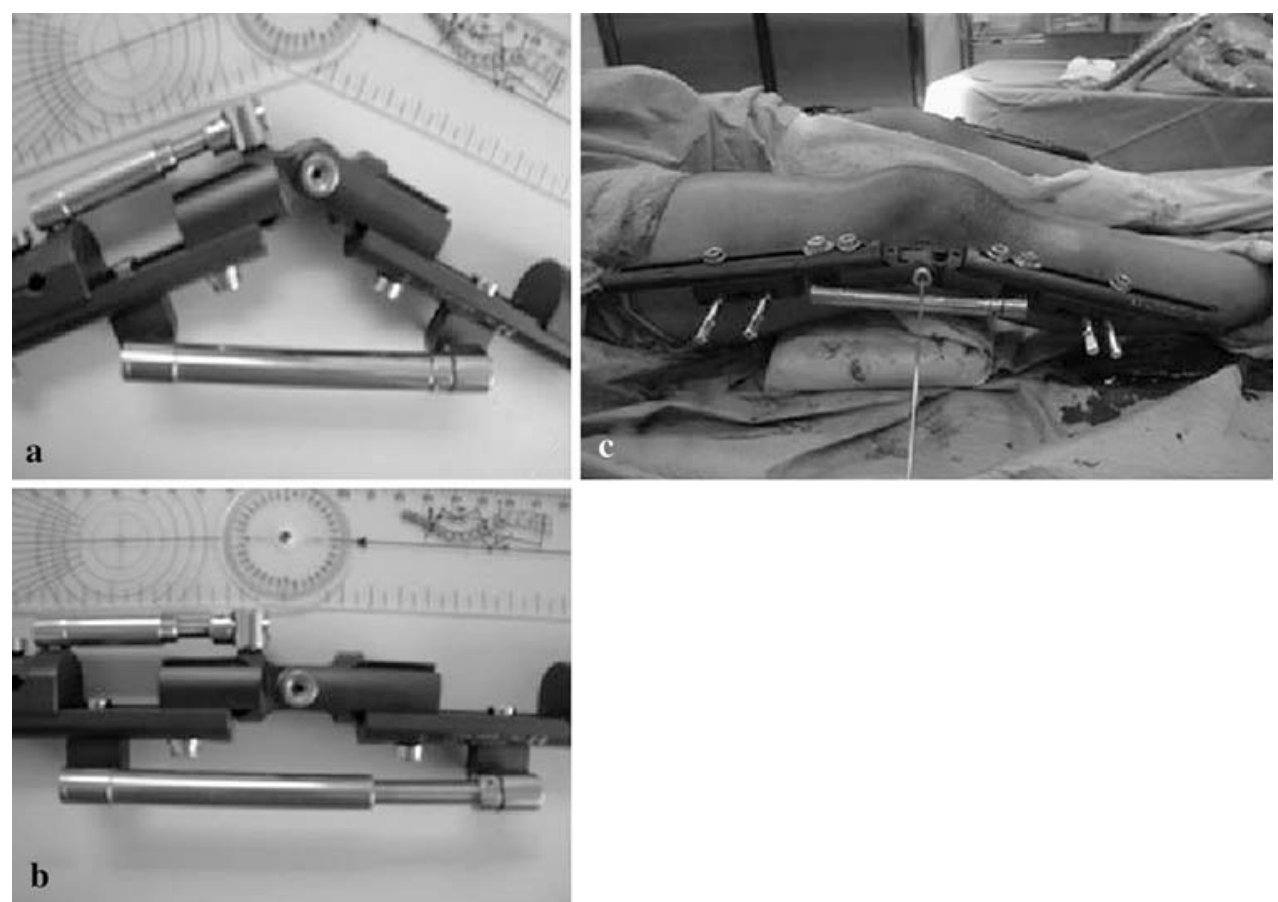

cadence and step length. This produces a crouched gait pattern with an excessive external foot progression angle, making it very difficult to lift their feet off the ground as a consequence of coincident flexion of the hip and rectus femoris spasticity $[2,4,9,10,13,18,23,24,32]$. Knee flexion contractures of more than $20-30^{\circ}$ are especially disabling, and calculations show that with $50-75^{\circ}$ contractures, these spastic patients must increase the strength of quadriceps action more than threefold in order to maintain balance during bipedestation [15, 24].

The treatment of knee flexion contractures should initially be physiotherapy and start as early as 5 years of age [21], but when contractures exceed $20-30^{\circ}$, in the absence of hip flexion deformity of more than $15^{\circ}$ and without spasticity in quadriceps, surgical options should be considered. The following surgical techniques have been used:

(1) Progressive serial corrective casts [8, 25],

(2) Bipolar traction of the extremity [28, 32],

(3) Soft tissue treatment techniques along with a variety of postoperative orthoses [2, 9, 11, 12, 14, 16, 25],

(4) Anterior femoral hemiepiphysiodesis [33] and

(5) Supracondylar femoral extension osteotomies [27, 34, 35].

The last two options are currently the most popular. Supracondylar femoral osteotomies have several disadvantages including: (a) spurious correction of the contracture through producing deformity in the distal femur-weightbearing then occurs mainly through the posterior femoral condyles and there is also a change in the orientation of the distal femoral physis to the floor; (b) the effect of the osteotomy is neutralized at a rate of $1 \%$ month because of rapid growth in small children and; (c) excessive articular compression is possible which could cause cartilage injuries of the femur and tibia $[34,35]$.

Hamstring lengthening is indicated in children under $7-8$ years of age with a flexion deformity greater than $30^{\circ}$ in order to achieve the best walking, standing, and sitting balance optimal for this age $[9,21]$. Post-operative immobilization methods vary from using casts for 2-7 weeks $[2,9,11,16,18,24]$ to progressive elongation systems such as circular external fixators of the Ilizarov type [25, 27, 29, 30, 32, 36], or monolateral fixators such as the model used by our team $[25,28]$. The popliteal angle and knee range of motion, together with the functional changes to ambulant and non-ambulant patients, are described in the literature as parameters to monitor. Improvement of the popliteal angle may vary between 50 and $89 \%[9,11,14,16,23,25,27,35]$ although recurrence which is estimated at around 3\% year is common in 4-34\% of the patients during a 3-4 year follow-up period $[9,11]$. In this group, we achieved an average correction of the popliteal angle in the immediate postoperative period of $57 \pm 8^{\circ}$, an improvement of $86 \%$, with a relapse of $20^{\circ}$ at the end of the 2 year follow-up period. The final correction in this series became $64 \%$, similar to the findings reported in the literature $[9,11,14,16,23,25,27]$. Also similar was the loss of correction of knee flexion, which amounted to a maximum of $10^{\circ}$ in our series, and the straight-leg raising angle which improved $25^{\circ}$ overall. The majority remained 
Duncan-Ely test negative at $90^{\circ}$ knee flexion with the exception of one case $(11.1 \%)$.

Several reports record a functional improvement in 31 to $70 \%$ of the patients [7, 11, 25, 28]. In this series, improvement occurred in $33 \%$ of the cases. Published results for correction of knee flexion contractures, irrespective of method, were excellent or good in $30-69 \%$ of the cases, average in $14-60 \%$ of the cases, and unsatisfactory in $10-21 \%$ of the cases [20, 25, 27]. These results are similar to ours: $56 \%$ excellent and good, $33 \%$ average, and $11 \%$ unsatisfactory. Genu recurvatum occurs in $4-16 \%$ after hamstring lengthening but seldom has a significant functional effect as it does not usually exceed $5-10^{\circ}$ and also improves with time $[9,11,16,24]$. In our study, $25 \%$ of our patient population experienced genu recurvatum. This high percentage was due to the association, in some cases, of gastrocnemius and distal hamstring lengthening and posterior capsulotomies. Nevertheless, and in agreement with other authors, we do not consider this evidence of a functional repercussion because it does not exceed $10^{\circ}$ $[9,24]$. Another complication we encountered was femorotibial arthrodiatasis which took place through technical error when applying joint distraction instead of extension; it is mentioned by other authors in up to $12 \%$ of the cases $[20,27]$, and we observed that this is totally reversible and it does not cause permanent sequelae.

No hypertension crises took place among our patients even though $78 \%$ of them underwent bilateral, simultaneous procedures. We believe that slow and progressive elongation through external fixation helps avoid the sudden stretching of the sympathetic nerves located around the blood vessels, which are believed to be the main etiology of this complication [36, 37]. We observed pin track infections in $69 \%$ of our cases, which is not very different from the percentage (between 30 and $83 \%$ ) reflected in the bibliography [28-30, 32]. This problem was controlled with local care and in only three cases were oral antibiotics required. The percentage of neurovascular or cutaneous complications, although not very high $(2.1-7.6 \%)$, is more likely if full extension is performed immediately at surgery in order to put on a cast or after supracondylar femoral osteotomies [34, 35]. It appears that the sciatic nerve does not tolerate elongations, especially when abruptly applied, of more than $5-12 \%$ of its length without injury [20, 38]. This has prompted the use of Ilizarov external fixation systems with progressive correction mechanisms as an alternative strategy [27, 29, 30, 32]. Published results are good but include miscellaneous pathologies such as congenital pterygium, arthrogryposis, haematological or septic problems, myelomeningocele, burns, and tumours. This Ilizarov method is technically demanding with a long learning curve $[28,29,36]$. The monolateral external fixator we used is not new and the technique has been described by other authors [25, 28, 31] but the samples in these reports are heterogeneous. Our results reported here compare favourably to those obtained using other techniques but the small sample size and short follow-up period necessitates we recommend caution when drawing conclusions and make it necessary to subject this technique to further evaluation. Nevertheless, we believe this technique to be versatile, easy to apply, generally well accepted by patients and their families, with a short learning curve, and effective according to the initial results obtained. For this reason we recommend its use for spastic paraplegic and quadriplegic children with knee flexion contractures greater than $30^{\circ}$ that have not responded with physiotherapy.

\section{References}

1. Grant A, Joy M-T, ÓBrien N, Hennessy E, MacDonald D (1989) Cerebral palsy among children born during the Dublin randomised trial of intrapartum monitoring. Lancet 25:1233-1235

2. Herring JA (2002) Disorders of the brain. In: Herring JA (eds) Tachdjian's pediatric orthopaedics from the Texas Scottish Rite Hospital for children. WB Saunders, Philadelphia, pp 1121-1242

3. Stanley FJ, Watson L (1992) Trends in perinatal mortality and cerebral palsy in Western Australia, 1967 to 1985. BMJ 304:1658-1663

4. Abel MF, Damiano DL, Pannunzio M, Bush J (1999) Muscletendon surgery in diplegic cerebral palsy: functional and mechanical changes. J Pediatr Orthop 19:366-375

5. Alberman E, Benson J, McDonald A (1982) Cerebral palsy and severe educational subnormality in low-birthweight children: a comparison of births in 1951-53 and 1970-73. Lancet 1(8272):606-608

6. Murphy DJ, Sellers S, MacKenzie IZ, Yudkin PL, Johnson AM (1995) Case-control study of antenatal and intrapartum risk factors for cerebral palsy in very preterm singleton babies. Lancet 346:1449-1454

7. Damron TA, Breed AL, Cook T (1993) Diminished knee flexion after hamstring surgery in cerebral palsy patients: prevalence and severity. J Pediatr Orthop 13:188-191

8. Brouwer B, Davidson LK, Olney SJ (2000) Serial casting in idiophatic toewalkers and children with spastic cerebral palsy. J Pediatr Orthop 20:221-225

9. Dhawlikar SH, Root L, Mann RL (1992) Distal lengthening of the hamstrings in patients who have cerebral palsy. J Bone Joint Surg Am 74:1385-1391

10. Hoffinger SA, Rab GT, Abou-Ghaida H (1993) Hamstrings in cerebral palsy crouch gait. J Pediatr Orthop 13:722-726

11. Damron T, Breed AL, Roecker E (1991) Hamstring tenotomies in cerebral palsy: long-term retrospective analysis. J Pediatr Orthop 11:514-519

12. Gage JR (1990) Surgical treatment of knee dysfunction in cerebral palsy. Clin Orthop 253:45-54

13. Hadley N, Chambers C, Scarborough N, Cain T, Rossi D (1992) Knee motion following multiple soft-tissue releases in ambulatory patients with cerebral palsy. J Pediatr Orthop 13:324-328

14. Marshall PD, Broughton NS, Menelaus MB, Graham HK (1996) Surgical release of knee flexion contractures in myelomeningocele. J Bone Joint Surg Br 78:912-916

15. Perry J, Antonelli D, Ford W (1975) Analysis of knee-joint forces during flexed-knee stance. J Bone Joint Surg Am 57:961-967 
16. Grujic H, Aparisi $\mathrm{T}$ (1982) Distal hamstring tendon release in knee flexion deformity. Int Orthop 6:103-106

17. Okawa A, Kajiura I, Hiroshima K (1990) Physical therapeutic and surgical management in spastic diplegia. A Japanese experience. Clin Orthop 253:38-44

18. Reimers J (1974) Contracture of the hamstrings in spastic cerebral palsy. A study of three methods of operative correction. J Bone Joint Surg Br 56:102-109

19. Sutherland DH, Santi M, Abel MF (1990) Treatment of stiff-knee gait in cerebral palsy: a comparison by gait analysis of distal rectus femoris transfer versus proximal rectus release. J Pediatr Orthop 10:433-441

20. Rodríguez-Merchán EC, Magallón M, Galindo E, López-Cabarcos C (1997) Hamstring release for fixed knee flexion contracture in hemophilia. Clin Orthop 343:63-67

21. Hoffer MM, Feiwell E, Perry R, Perry J, Bonnett C (1973) Functional ambulation in patients with myelomeningocele. J Bone Joint Surg Am 55A:137-148

22. Lieber RL (1994) Experimental design and statistical analysis. In: Simon SR (eds) Orthopaedic basic science. American Academy of Orthopaedic Surgeons, Columbus, pp 623-665

23. Reimers J (1990) Functional changes in the antagonists after lengthening the agonist in cerebral palsy. II. Quadriceps strength before and after distal hamstring lengthening. Clin Orthop 253:35-37

24. Thometz J, Simon S, Rosenthal R (1989) The effect on gait of lengthening of the medial hamstrings in cerebral palsy. J Bone Joint Surg Am 71:345-353

25. Herzenberg JE, Davis JR, Paley D, Bhave A (1994) Mechanical distraction for treatment of severe knee flexion contractures. Clin Orthop 301:80-88

26. Hollister AM, Jatana S, Singh AK, Sullivan WW, Lupichuk AG (1993) The axes of rotation of the knee. Clin Orthop 290:259-268

27. Huang SC (1996) Soft tissue contractures of the knee or ankle treated by the Ilizarov technique. Hugh recurrence rate in 26 patients followed for 3-6 years. Acta Orthop Scand 67:443449

28. Mooney JF 3rd, Koman LA (2001) Knee flexion contractures: soft tissue correction with monolateral external fixation. J South Orthop Assoc 10:32-36

29. Volkov MV, Oganesian OV (1975) Restoration of function of the knee and elbow with a hinge-distractor apparatus. J Bone Joint Surg Am 57:591-599

30. Gillen JA 2nd, Walker JL, Burgess RC, Stevens DB (1996) Use of Ilizarov external fixator to treat joint pterygia. J Pediatr Orthop $16: 430-437$

31. Herzenberg JE, Waanders NA (1991) Calculating rate and duration of distraction for deformity correction with the Ilizarov technique. Orthop Clin North Am 22:601-611

32. Damsin JP, Ghanem I (1996) Treatment of severe flexion deformity of the knee in children and adolescents using the Ilizarov technique. J Bone Joint Surg Br 78:140-144

33. Kramer A, Stevens PM (2001) Anterior femoral stapling. J Pediatr Orthop 21:804-807

34. Asirvatham R, Mukherjee A, Agarwal S, Rooney RJ, Ellis RD, Watts HG (1993) Supracondylar femoral extension osteotomy: its complications. J Pediatr Orthop 13:642-645

35. Del Bello DA, Watts HG (1996) Distal extension osteotomy for knee flexion contracture in patients with arthrogryposis. J Pediatr Orthop 16:122-126

36. Damsin JP, Carlioz H (1994) Traitement des deformations des membres par la méthode d́llizarov. Rev Chir Orthop Reparatrice Appar Mot 80:324-333

37. Shah A, Asirvatham R (1994) Hypertension after surgical release for flexion contractures of the knee. J Bone Joint Surg Br 76:274277

38. Aspden RM, Porter RW (1994) Nerve traction during correction of knee flexion deformity. A case report and calculation. J Bone Joint Surg Br 76:471-473 